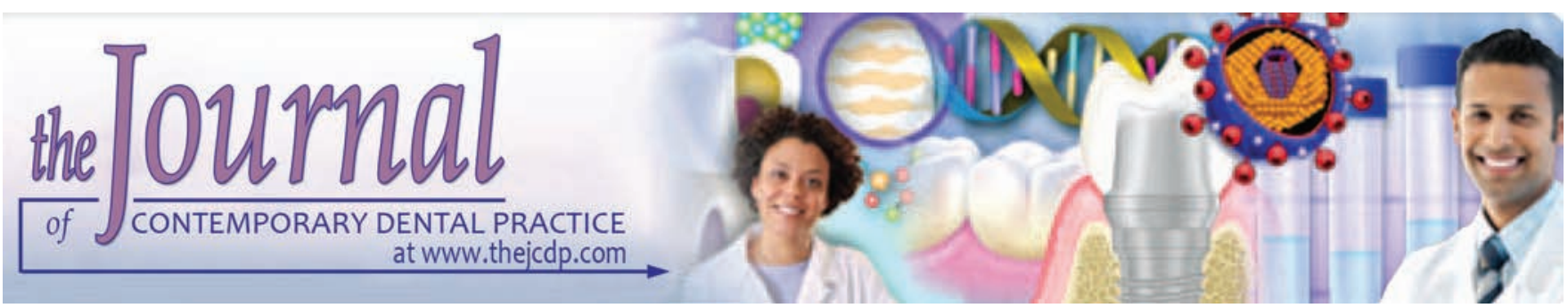

\title{
Evaluation to prevent the Physical Changes in Colored Elastomeric Modules when exposed to various Dietary Media
}

${ }^{1} \mathrm{~S}$ Dhivya Kanya, ${ }^{2} \mathrm{~K}$ Pradeep Babu, ${ }^{3} \mathrm{R}$ Venkatesan, ${ }^{4} \mathrm{~A}$ Nanda Kumar

\begin{abstract}
Aim and objective: The aim of the study is to analyze and compare the variation of lumen size and thickness of the elastomeric colored modules when immersed in four selected dietary media.
\end{abstract}

Materials and methods: Sample size of 40 modules on each color - such as red, blue, green, and black - was taken and immersed in four dietary media (artificial saliva, coke mixed with artificial saliva, turmeric mixed with artificial saliva, and coffee mixed with artificial saliva). Beakers containing different dietary media and color modules are kept in the incubator at $37^{\circ} \mathrm{C}$ for 72 hours. After incubation period, all the segments of module strips removed from the dietary media were placed under the electric lamp and photographs were taken using Canon camera (SX400 IS). Photographs were transferred to GIMP software, and lumen size and thickness variation in the modules was measured.

Results: Statistical analysis were performed using analysis of variance and t-test in Statistical Package for the Social Sciences software. It showed significant difference in thickness of black module in all dietary media. Significant difference existed between all the lumen sizes of four color modules in four dietary media.

Conclusion: This study was done to identify the material that has more changes in physical properties when exposed to various dietary media.

\footnotetext{
${ }^{1}$ Department of Orthodontics and Dentofacial Orthopedics, Sri Ramakrishna Dental College and Hospital, Coimbatore, Tamil Nadu, India

${ }^{2}$ Department of Orthodontics and Dentofacial Orthopedics, Indira Gandhi Institute of Dental Sciences, Puducherry, India

${ }^{3}$ Private Dental Clinic, Coimbatore, Tamil Nadu, India

${ }^{4}$ Department of Orthodontics and Dentofacial Orthopedics Meenakshi Ammal Dental College, Chennai, Tamil Nadu India

Corresponding Author: S Dhivya Kanya, Department of Orthodontics and Dentofacial Orthopedics, Sri Ramakrishna Dental College and Hospital, Coimbatore, Tamil Nadu, India Phone: +919443007701, e-mail: dhivyakanya@gmail.com
}

Clinical significance: According to the results obtained, black color modules have increased in lumen size in all dietary media. In thickness, red color module showed less variation and black color module exhibited more variation.

Keywords: Artificial saliva, Digital camera, Inch, Medium, Modules, Photograph.

How to cite this article: Kanya SD, Babu KP, Venkatesan R, Kumar AN. Evaluation to prevent the Physical Changes in Colored Elastomeric Modules when exposed to various Dietary Media. J Contemp Dent Pract 2017;18(6):458-462.

Source of support: Nil

Conflict of interest: None

\section{INTRODUCTION}

Advances in orthodontic treatment have helped the clinician to effectively treat the patient. Newly introduced materials satisfy the patient esthetically during the treatment. Modules after being introduced have gained instant popularity because of its use and reduction in chair-side time. ${ }^{1}$

Even though the physical properties of these color modules are tested before clinical use, orthodontists have found minor physical changes in the color modules in the oral environment, especially in the case of physical properties, such as content of color and dimension. The dimensional changes, such as variation in the thickness and lumen size, are the factors to be noted, especially when it is incorporated in various dietary media. These variations can interrupt the light continuous force produced by them.

\section{Aim}

The aim of the study was to analyze the lumen size and thickness of different color elastomeric modules by exposing them to various dietary media. ${ }^{2}$ 

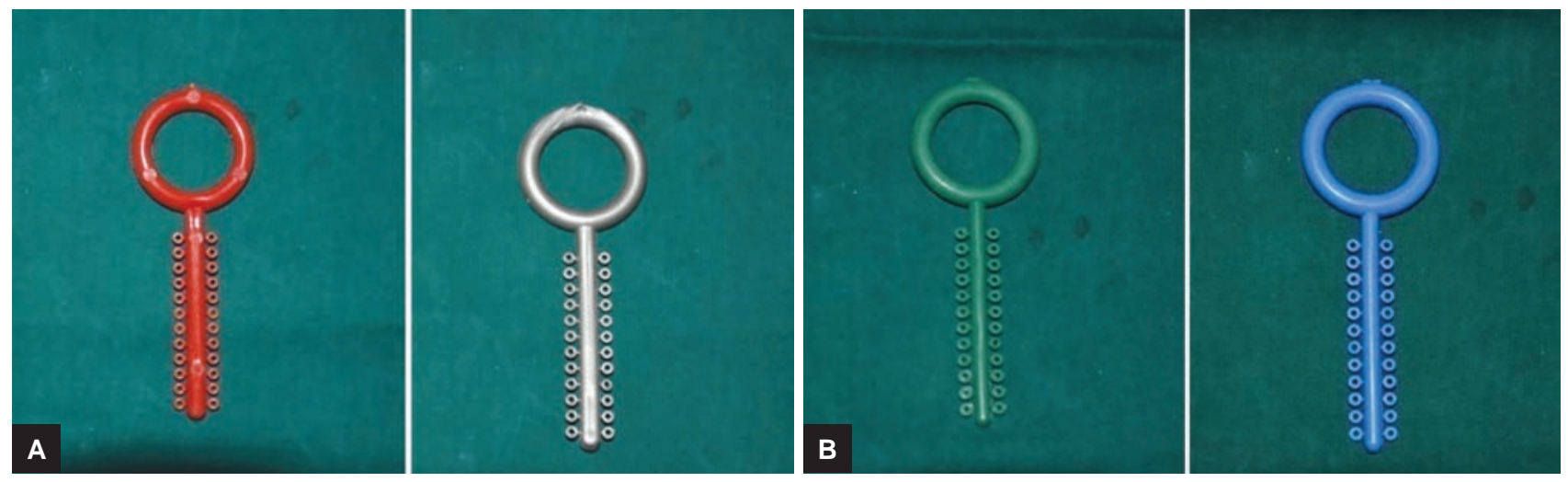

Figs $1 \mathrm{~A}$ and $\mathrm{B}$ : Color modules

\section{MATERIALS AND METHODS}

Four dietary media, such as artificial saliva, coke mixed with artificial saliva, turmeric mixed with artificial saliva, and coffee mixed with artificial saliva are equally divided into four parts of $25 \mathrm{~mL}$ for each color module. ${ }^{3}$ Color modules were procured (blue, black, green, and red) (Fig. 1) from American Orthodontics of 160 numbers totally, and each color module was immersed in four dietary media. All beakers were placed in an incubator at $37^{\circ} \mathrm{C}$ for 72 hours (Fig. 2). After the incubation period, modules were removed and placed under the electric lamp and photographs were taken using Canon 20 megapixel camera (SX400 IS). The camera was placed at a distance of one foot on a stable wooden box. Photographs were taken (Fig. 3) with 20 times magnification. ${ }^{4}$ Photographs were transferred to the computer and using the GIMP software, ${ }^{5}$ inner diameter and the outer diameter were measured to determine the lumen size and thickness variation in the modules. In this study, the unit of measurement of lumen size and thickness was obtained in inches (Fig. 4).

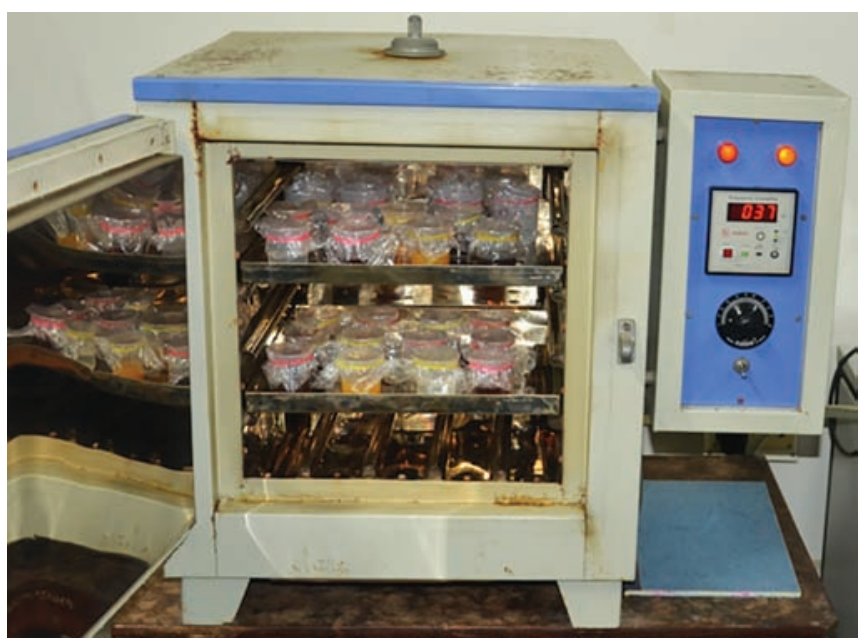

Fig. 2: Samples in incubator at $37^{\circ} \mathrm{C}$

\section{RESULTS}

Important significance existed between the lumen size of modules when immersed in various dietary media, with $p<0.05$. The black color module lumen size is increased in
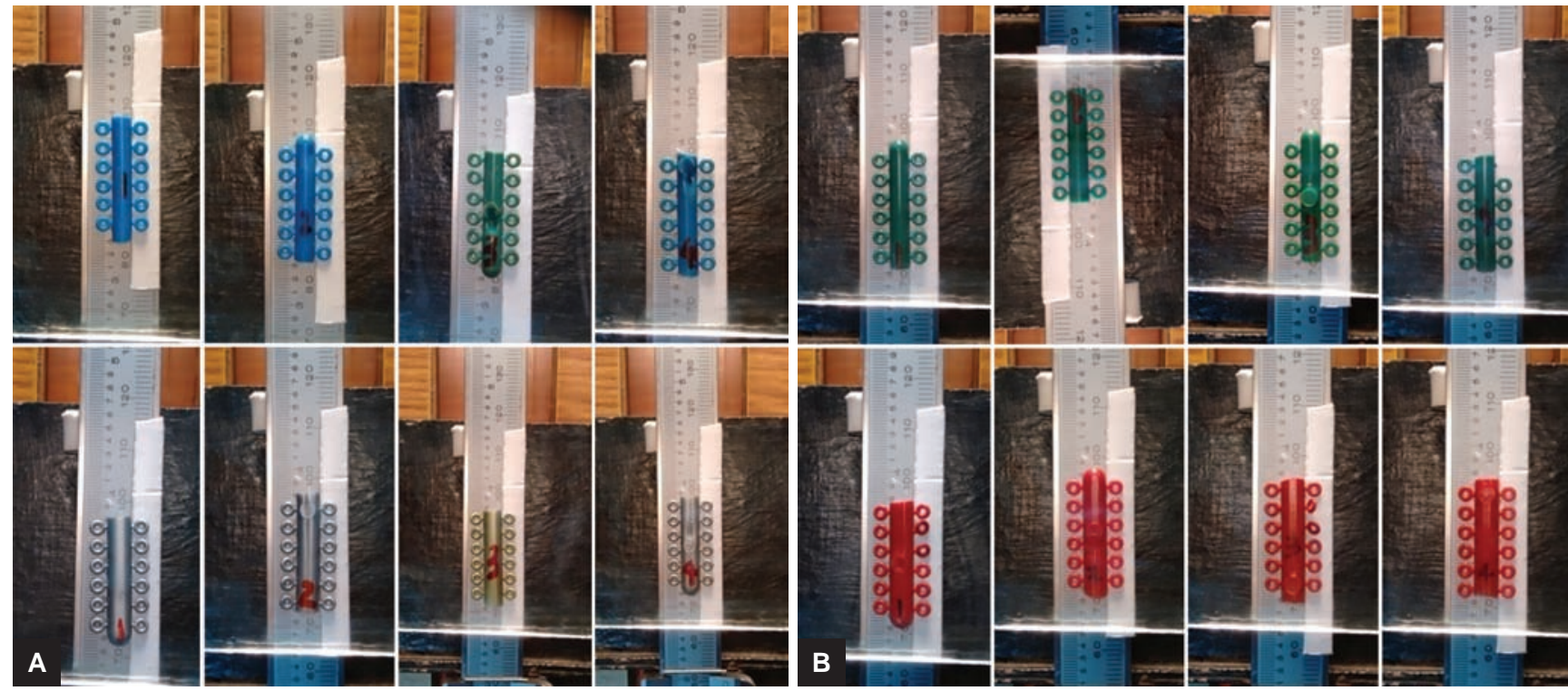

Figs $3 \mathrm{~A}$ and $\mathrm{B}$ : Images obtained using digital camera 


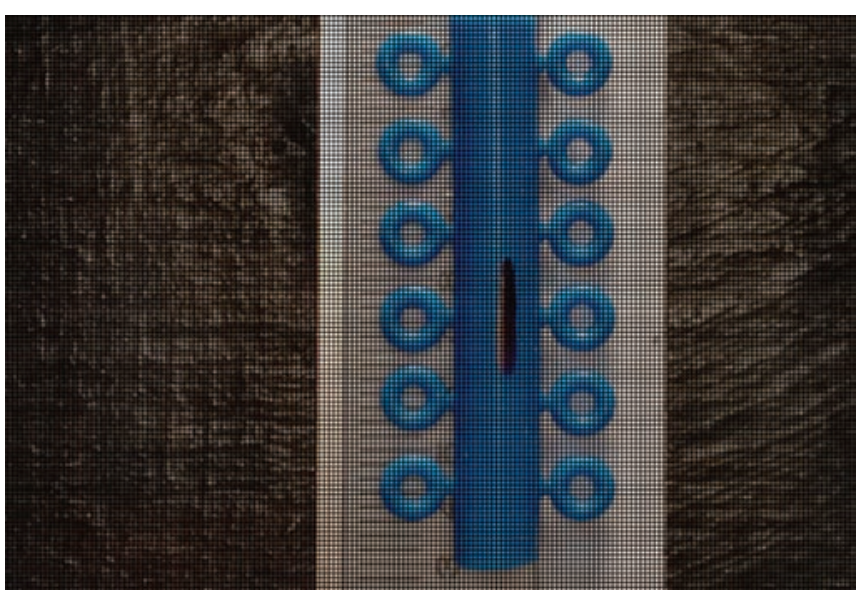

Fig. 4: Analysis using GIMP software

the medium of coke mixed with artificial saliva compared with lumen size of other samples (Graph 1). A significant difference existed between the thicknesses of modules when immersed in various dietary media, with $\mathrm{p}<0.05$. Black color module thickness is more reduced in all dietary medium compared with other samples (Graph 2).

\section{DISCUSSION}

GIMP software analyzed black color modules immersed in artificial saliva showed more variation in the lumen size compared with the other color modules (blue, green, and red). Lumen size of the black color modules increased when compared with the standard lumen size by 0.003 inch. This increase in lumen size of the black color module may be utilized to the advantage by using these modules in friction mechanism, where the wire passes through the bracket slot to induce tooth movement. Lumen size of the red color module has decreased when compared with the standard lumen size by 0.001 inch, which is less compared with the other modules. Since the red color module lumen size is decreased compared with

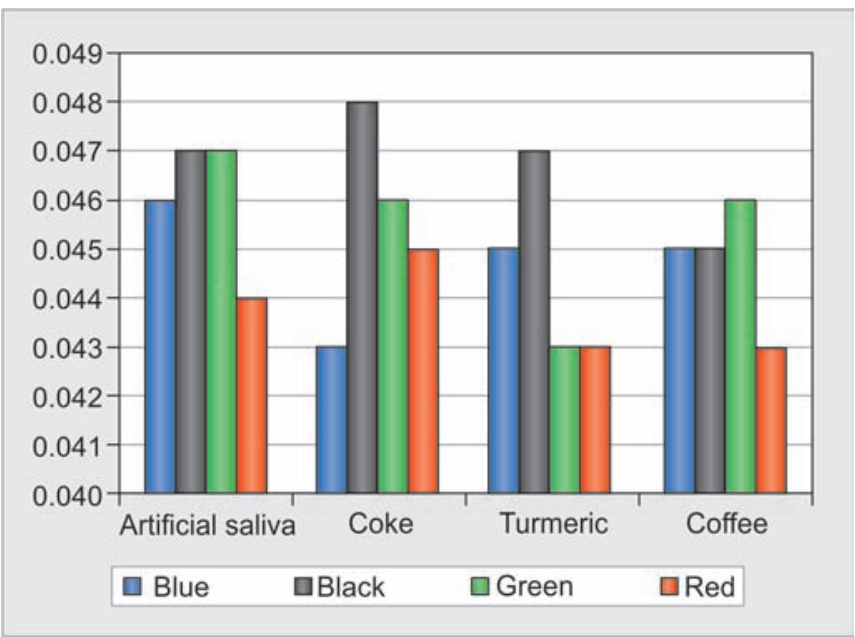

Graph 1: Lumen size of colored modules after being exposed to various dietary media the standard value, clinically we can assume that more force is generated in the bracket wire interface to achieve maximum results and to reduce treatment duration. ${ }^{6}$ Blue color module lumen size is increased by 0.001 inch than the standard lumen size, and green color module is 0.002 inch than standard lumen size. von Fraunhofer et $\mathrm{al}^{7}$ described that the artificial saliva affects the elastic property. Similar to artificial salivary medium, coke mixed with artificial saliva also shows significant variation in the lumen size of black color modules compared with other samples in this medium. The lumen size of black color modules in this medium is 0.048 inch, which is 0.003 inch more than the manufacturer standard value. Green color module lumen size in artificial saliva is 0.046 inch, which is increased by 0.001 inch compared with the standard value. Lumen size of the red color module in artificial salivary medium is maintained with same standardized lumen size of 0.045 inch. If unwanted tooth movement has to be avoided, red color module can be advised to the patient, whereas blue color module lumen size is 0.043 inch and it is reduced by 0.002 inch than the standard lumen size provided by the manufacturer. This decrease in lumen size of blue color modules exposed coke mixed with artificial saliva might be due to the $\mathrm{pH} 2.05$ of coke. Hence, the orthodontic patients consuming more quantity of coke will affect the lumen size of the blue color module by decreasing it by 0.003 inch; this alteration of lumen size of this module can be utilized in initial phases where arch wire should be completely adapted in the bracket slot for the correction of crowding as these modules generate comparatively more amount of force. Leandro et $\mathrm{al}^{8}$ determined that the coke solution is modifying the property of black color module. The third dietary media is the combination of turmeric with artificial saliva. Lumen size of black color module is increased by 0.002 inch than the standard value in this medium. There is no variation

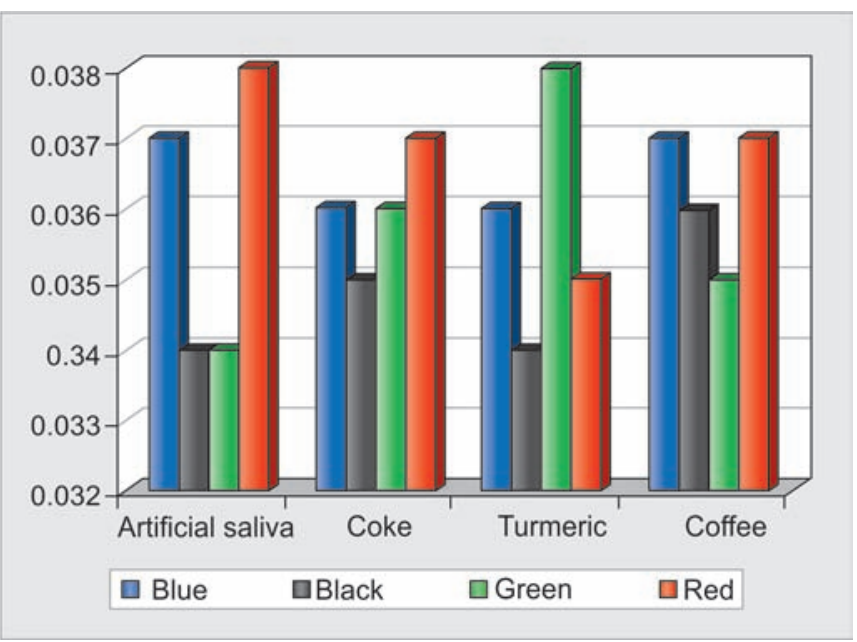

Graph 2: Thickness of colored modules after being exposing to various dietary media 
in the lumen size of blue color modules. These modules can be used as active lacebacks in baseline test mechanics for retraction. This might be due to $\mathrm{pH}$ of turmeric as there is no substantial difference in the lumen size. Lumen size variation of green and red color modules is same as 0.043 inch; these are 0.002 inch lesser than the standard lumen size. Red and green color modules can be advised for the patients, who add more amount of turmeric to their diet. These color modules can be utilized for active tie back during retraction phase. The use of turmeric as dietary media showed greater variation in the physical property of the elastomeric chain. ${ }^{5}$ Nattrass et $\mathrm{al}^{9}$ have analyzed the effect of environmental medium on color modules and showed that turmeric demonstrated the greatest force loss and this continued until the end of the 7 day test period.

Artificial saliva is mixed with coffee powder; green color modules have a lumen size of 0.046 inch, which is slightly higher than the standard value by 0.001 inch. In the diet chart of the patient, if frequency of coffee consumption is more, green color module can be advised for the patient to prevent friction resistance. Black and blue color modules have no alteration in the lumen size in this medium, and it is maintaining the standardized lumen size. According to the diet, blue or black color modules can be placed to avoid unwanted tooth movement. Red color module lumen size is 0.043 inch, which is reduced by 0.002 inch compared with the standard lumen size. Red color modules can be given in coffee-consuming patients in the initial aligning phase, but the periodontal condition of the patient should be observed as most of the patients are adults. Thickness evaluation of the color elastomeric module is determined after immersion of the colored modules in various dietary media for 72 hours at $37^{\circ} \mathrm{C}$. The increase in surface area, i.e., the thickness of the module can increase the microbial absorption and accumulation. The value of thickness is given by the manufacturer and reevaluated for this study and analyzed as $0.07 \mathrm{inch}$. According to the result obtained, the thickness of the colored modules is reduced compared with the manufacturer value, i.e., the thickness of colored modules is reduced after immersing in the dietary medium for 72 hours at $37^{\circ} \mathrm{C}$. Thickness of the elastomeric colored modules was analyzed after exposing to artificial salivary medium and compared with the standard value given by the manufacturer.

In the medium of artificial saliva, thickness variation of black color module is of 0.034 inch and, i.e., reduced by 0.036 inch than the standard value. Green color module thickness is also reduced by 0.036 inch compared with the manufacturer value. Thickness of blue color module is 0.037 inch in the medium of artificial saliva; this thickness is less than the normal value by 0.033 inch. Red color module thickness is 0.038 inch; the difference of this thickness from the standard value is by $0.032 \mathrm{inch}$. When the thickness is reduced, the complete engagement of wire in the bracket slot is not possible because force application on the wire is not adequate and the elasticity of the modules was decreased. The effects of reduction in thickness may lead to decrease in strength and elasticity and may ultimately lead to tear of modules. von Fraunhofer et $\mathrm{al}^{7}$ have evaluated the loss of elasticity of elastomeric chain in artificial saliva.

In the dietary medium of coke mixed with artificial saliva, thickness of the black color module is 0.035 inch; this value is less than the standard value by 0.035 inch. Blue color module thickness is 0.036 inch, which is reduced compared with the manufacturer value by 0.034 inch. Green color module has reduced thickness of 0.036 inch, and it is reduced by 0.034 inch than standard value. Thickness of red color module in this dietary media is 0.037 inch; this color module is also reduced than the manufacturer value by 0.033 inch. In the overall comparison of the thickness of color modules, it is determined that the black color module thickness is reduced more than the standard value. This variation in thickness may lead to tear of modules when used with heavy wires during retraction phase.

Leandro et $\mathrm{al}^{8}$ suggested that the $\mathrm{pH}$ of coke has modified the physical property of black color module. In the medium of artificial saliva with turmeric, black color module has the thickness of 0.034 inch, which is less than the standard value of $0.07 \mathrm{inch}$. The variation of thickness of the black color module is 0.036 inch. Thickness of blue color module is reduced to 0.036 inch from the standard value by 0.034 inch. Green color module has reduced the thickness to 0.038 inch, which is 0.032 inch less than the standard value. There is reduction in the thickness of red color modules in this medium to 0.035 inch and it has reduced by 0.035 inch compared with the standard value. Comparing all the four colors, black color module thickness is reduced more in this medium. When the thickness of color modules is reduced, less amount of microorganism gets accumulated, but the modules may not perform as prescribed by the manufacturer, even though the modules are not stretched there is a huge decrease in thickness; there might be more reduction in thickness when placed in the oral cavity. Turmeric showed greater variation in the physical property of the elastomeric chain. ${ }^{5}$ Nattrass et $\mathrm{al}^{9}$ analyzed the force loss of modules when tested in turmeric medium. Thickness of the green color module is $0.035 \mathrm{inch}$, and this value is decreased than the standard value by 0.035 inch when immersed in artificial saliva with coffee. Black color module thickness is reduced to 0.036 inch, which is less than the value provided by the manufacturer. The variation of black color module thickness from the standard 
value is 0.034 inch. Thickness of blue color module and red color module is reduced to $0.037 \mathrm{inch}$; the difference in the thickness of blue color module and red color module with the standard value is 0.033 inch. Red and green color module can be provided, in which thickness is not much reduced comparatively. Due to the severe reduction in thickness, elastic properties of the modules may be lost and is less effective to hold the wire in the bracket slot. Overall, evaluation of thickness of all four color modules in all the four dietary media showed a significant reduction in the thickness of the modules. These reductions in the thickness may cause loss of elasticity, which is a prime factor and force degradation. Due to this decrease in thickness, these may lead to tear of the module when heavy wires or heavy forces are applied on these modules. ${ }^{10}$

\section{CONCLUSION}

Knowledge of dietary intake of the orthodontic patient is very important as these dietary products influence the material characteristics used by the orthodontists in the oral cavity. Different orthodontic materials react unusually in the oral cavity to various dietary media. These products exhibit several physical and structural changes when subjected to various dietary media. These changes can influence the biomechanics directly or indirectly.

This study was done to identify the material which has more changes in physical properties when exposed to various dietary media. This study reveals that black color modules had a tendency for increasing lumen size in all the dietary media, whereas red color modules exhibited least deviation in all the four dietary media. In thickness variation, all the color modules showed a significant decrease in thickness, among those black color module expressed maximum decrease in thickness.

\section{REFERENCES}

1. Bortoly TG, Guerrero AP, Rached RN, Tanaka O, GuarizaFilho O, Rosa EA. Sliding resistance with esthetic ligatures: an in-vitro study. Am J Orthod Dentofacial Orthop 2008 Mar;133(3):340.e1-340.e7.

2. Kanya SD, Babu KP, Hanumanth S, Kumar MS, Kumar AN, Venkatesan, Mathew A, Priya S, Yashwant A, Arvinth. Evaluation of colored elastomeric modules for leaching properties when exposed to various dietary media: an in vitro study. J Int Oral Health 2016 Jan;8(1):58-62.

3. Ardeshna AP, Vaidyanathan TK. Color changes of orthodontic elastomeric module materials exposed to in vitro dietary media. J Orthod 2009 Sep;36(3):177-185.

4. Kim SH, Lee YK. Measurement of discoloration of orthodontic elastomeric modules with a digital camera. Am J Orthod Dentofacial Orthop 2009 Oct;31(5):556-562.

5. Babaloukas G, Tentolouris N, Liatis S, Sklavounou A, Perrea D. Evaluation of three methods for retrospective correction of vignetting on medical microscopy images utilizing two open sources software tools. J Microsc 2011 Aug;244(Pt 3):320-324.

6. Ash JL, Nikolai RJ. Relaxation of orthodontic elastomeric chains and modules in vitro and in vivo. J Dent Res 1978 May-Jun;57(5-6):685-690.

7. von Fraunhofer JA, Coffelt MT, Orbell GM. The effects of artificial saliva and topical fluoride treatments on the degradation of the elastic properties of orthodontic chains. Angle Orthod 1992 Winter;62(4):265-274.

8. Leandro teixeria et al. The Environmental Influence of Light Coke, Phosphoric Acid, and Citric Acid on Elastomeric Chains. J Contemp Dent Prac 2008; 9(7):1-9.

9. Nattrass C, Ireland AJ, Sherriff M. The effect of environmental factors on elastomeric chain and nickel titanium coil springs. Eur J Orthod 1998 Apr;20(2):169-176.

10. BalhoffDA,Shuldberg M,HaganJL, Ballard RW, Armbruster PC. Force decay of elastomeric chains - a mechanical design and product comparison study. J Orthod 2011 Mar;38(1):40-47. 